First publ. in: Physical Review A 7 (1973), 3, pp.1130-1134

\title{
Sound-Modulated Flow of Superfluid Helium through a Small Orifice: An Attempt to Observe the ac Josephson Effect*
}

\author{
P. Leiderer and F. Pobell ${ }^{\dagger}$ \\ Physik-Department, Technische Universität, München, Germany \\ (Received 2 October 1972)
}

\begin{abstract}
Two chambers filled with superfluid helium to different levels were connected by a small orifice $(10-15 \mu \mathrm{m})$. The flow of the liquid through the orifice was modulated by a sound field. With one experimental apparatus only a continuous flow was observed. With a modified apparatus it was possible to obtain stable states with no net flow at finite level differences between the baths. The spacing between these states is inversely proportional to the sound frequency. It depends on the total height of the liquid in one of the baths and not on the level difference between them. We conclude that the stable states of zero flow result from ultrasonic standing waves of the superfluid in one of the chambers. A phase coupling or Josephson effect can be excluded as an explanation for these states.
\end{abstract}

\section{INTRODUCTION}

One of the most fascinating aspects about superfluid helium is the similarity of many of its properties to the properties of metallic superconductors. The many analogies between these superfluids suggest the possibility of observing the ac Josephson effect in Herr. Experiments whose results were interpreted as the ac Josephson effect in superfluid helium were reported some years ago in Refs. 1 and 2 , and recently in Ref. 3. In these experiments two chambers filled with superfluid helium to different levels were connected by a small orifice, as shown in Fig. 1. Although this orifice is very large (about $10 \mu \mathrm{m}$ ) compared to the coherence length of He II it is supposed to act as a "weak link" between the two baths. Below the orifice an ultrasonic transducer is mounted. Its sound field can modulate the flow of superfluid through the orifice. The authors ${ }^{1-3}$ observed interruption of flow at distinct level differences $\Delta z$ between the two baths. The arrests seem to occur whenever

$$
n_{1} m g \Delta z=n_{2} h \nu,
$$

where $n_{1}$ and $n_{2}$ are integers, $m$ the mass of a ${ }^{4} \mathrm{He}$ atom, $g$ the gravitational acceleration, $\Delta z$ the level difference, $h$ Planck's constant, and $\nu$ the frequency of sound.

This Josephson frequency relation proposed by Anderson ${ }^{4}$ is based on two concepts: (i) There is a phase slippage of the superfluid order parameters of the two coupled baths due to a chemical potential difference between them. This difference results from the difference $m g \Delta z$ in gravitational potential. (ii) The variation of the phase may be synchronized by an external sound field of frequency $\nu$. Then the net flow between the two baths may be interrupted whenever the difference in gravitational potential is compensated by a constant phase slippage. This phase slippage could result from the generation and motion of vortices in the orifice at a rate $\left(n_{2} / n_{1}\right) \nu$. Details can be found in Refs. 1-4. Several aspects of these experiments have remained unexplained, particularly with regard to what is . happening at the orifice. In addition, steps in the flow curve have only been observed when the difference in the chemical potential was created by a gravitational head difference. ${ }^{5}$

In 1968 we tried to repeat and possibly extend the above-mentioned experiments by varying some ex- 


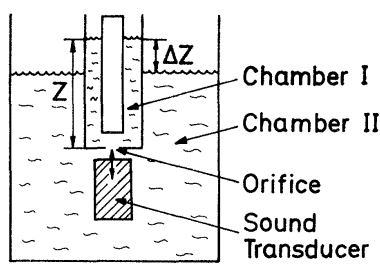

FIG. 1. Schematic diagram of the apparatus used to investigate the ac Josephson effect in He II. Helium flow through the orifice is modulated by an ultrasonic transducer.

perimental parameters. ${ }^{6}$ Two experimental setups were used which were very similar to those of the other groups. ${ }^{1-3}$ With one arrangement we were unable to produce any steps or irregularities in the flow of He II through the orifice, either with or without a superimposed sound field. With the second setup we observed steps in the flow of the superfluid when sound was switched on. These steps were as well defined and stable as in the experiments of Khorana and Chandrasekhar, ${ }^{2}$ for example. But by performing several checks we could exclude any phase coupling or Josephson effect as an explanation for our observations. Instead, we can show that the steps resulted from ultrasonic standing waves in the superfluid.

After completing the experiments in 1968 the results were not published but communicated to some groups possibly interested in them. In the meantime other groups have also been unable to observe the ac Josephson effect in He Ir. ${ }^{7}$ This somewhat obscure situation and the great interest it received at a discussion seminar at the recent LT XIII conference ${ }^{8}$ encouraged us to publish our observations now.

\section{EXPERIMENTAL ARRANGEMENTS}

\section{A. Apparatus 1}

Our first apparatus is shown in Fig. 2. It is very similar to the ones of Refs. 1-3. Chamber I was a coaxial capacitor used to monitor the liquid level. The capacitor was part of a tunneldiode oscillator. By monitoring the frequency on a chart recorder, the liquid level in the capacitor could be determined to $\pm 0.01 \mathrm{~mm}$. The liquid level in chamber II could be observed in a glass capillary. This level remained essentially constant during an experiment because the free cross section of the capillary is large compared to that of the capacitor. The two chambers were connected only by a $10-\mu \mathrm{m}$-diam orifice punched into a $50-\mu \mathrm{m}-$ thick $\mathrm{Cu}$ foil. This foil was glued to a 1-mm-thick brass plate (containing a wider hole) to prevent it from vibrating. An ultrasonic transducer was placed about $0.1 \mathrm{~mm}$ below the foil in the lower chamber. It was supported by its electrical leads. Quartz as well as PXE ceramic transducers were used to provide the sound field. When this transducer was switched off, an identical dummy trans-

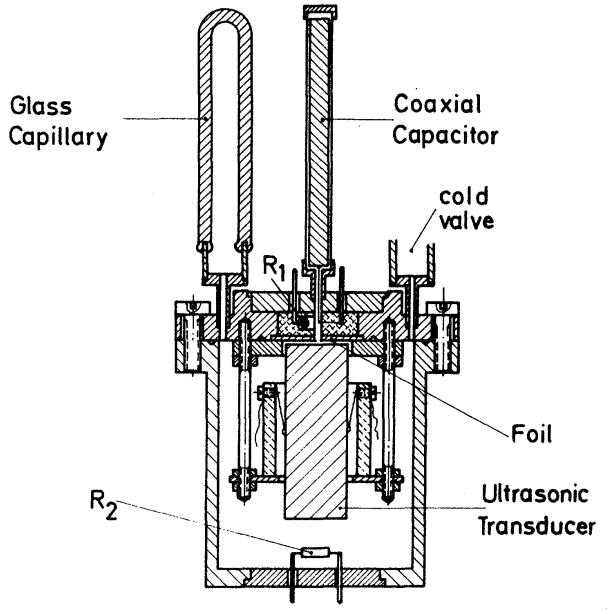

FIG. 2. Apparatus 1: The Cu foil $(50 \mu \mathrm{m}$ thick; $10-$ $\mu \mathrm{m}$ orifice) was glued to a $1-\mathrm{mm}$-thick brass plate; $R_{1}$, $R_{2}$ : carbon thermometers. The coaxial capacitor (o.d. $=2.0 \mathrm{~mm}$; i. d. $=1.9 \mathrm{~mm}$ ) was $4.0 \mathrm{~cm}$ long.

ducer in chamber II was turned on to keep the heat input constant. Temperature differences were measured in both chambers with carbon resistors to $50 \mu \mathrm{K}$. At the beginning of an experiment the chambers were about half filled with liquid helium from the main Dewar. Then the cold valve was closed to seal off the system. The cryostat used for the experiments was vibration isolated with a frequency cutoff of about $1 \mathrm{~Hz}$.

\section{B. Apparatus 2}

In other experiments the simpler apparatus shown in Fig. 3 was used. Here a glass capillary with an inner diameter of $1.5 \mathrm{~mm}$ served as chamber I. The level of the liquid in it was measured optically with a cathetometer to within $\pm 0.02 \mathrm{~mm}$. The liquid helium in the main Dewar served as the second superfluid bath. In this apparatus another $50-\mu \mathrm{m}$-thick $\mathrm{Cu}$ foil with a $15-\mu \mathrm{m}$-diam orifice connected the two baths. This foil was also glued to a $1-\mathrm{mm}$-thick brass plate with a wider hole in it to:

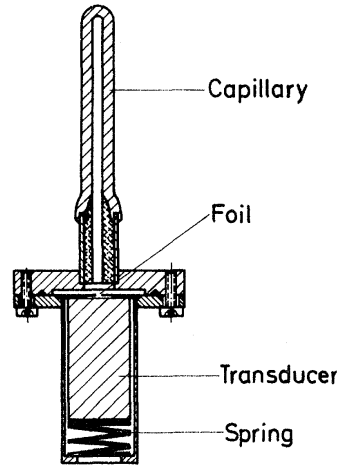

FIG. 3. Apparatus 2: The glass capillary had an i.d. of $1.5 \mathrm{~mm}$. For the experiments discussed in Sec. III C the 1-mm-thick brass plate to which the $\mathrm{Cu}$ foil $(50 \mu \mathrm{m}$ thick; $15-\mu \mathrm{m}$ orifice) was glued was re-moved. 
prevent vibrations of the foil. Removing this stabilizing plate had a significant influence on the experimental results, as will be discussed in Sec. IIIC. The ultrasonic transducer was about $0.05 \mathrm{~mm}$ below the foil and was held in place by a spring. This setup was much more exposed to external disturbances than apparatus 1 .

\section{RESULTS AND DISCUSSION}

\section{A. Pumping Action of Transducer}

Liquid helium was pumped out of chamber I by switching on the transducer, resulting in a difference in the liquid level in the two chambers. We needed a higher transducer power than reported by Khorana ${ }^{2}$ to reach the same level difference. But this might be insignificant because the pumping action depended strongly on geometry. The pumping efficiency of the transducers was the same at 1.3 and $1.8 \mathrm{~K}$. One of the striking features was a considerably reduced efficiency of a transducer for pumping at temperatures above $T_{\lambda \cdot}{ }^{9}$ These results are shown in Fig. 4.

\section{B. Flow Curves with Fixed Foil}

Without a superimposed sound field, the velocity for gravitational flow of the superfluid through the orifice was the same for both flow directions and was slightly dependent on the level difference (see Fig。5)。

As mentioned in Sec. II, the foil was glued to a metal plate to prevent it from vibrating due to the sound field. Then for both experimental setups we were unable to detect any steps or irregularities in the flow of He II through the orifice with or without the ultrasonic field superimposed. An example of the observations of this smooth flow with apparatus 1 is shown in Fig. 5. The liquid levels were in equilibrium before the transducer was turned on. After switching on the transducer (ceramic, 102 $\mathrm{kHz}$ ), liquid was pumped out of the capacitor above the foil. The second part of the figure shows the free backflow of the superfluid with a critical ve-

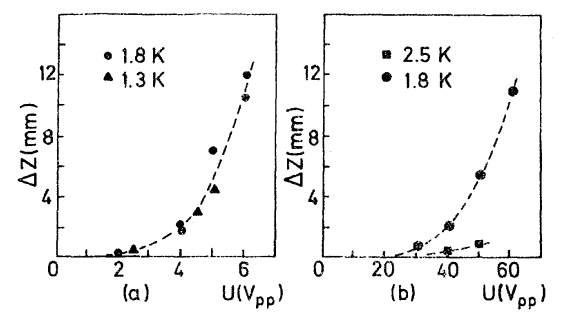

FIG. 4. Pumping action of the transducers: the level difference $\Delta z$ between the two baths created by the transducers excited with the peak volitage $U$. (a) Ceramic transducer: $\nu=1.02 \mathrm{kHz}, T=1.3$ and $1.8 \mathrm{~K}$. (b) Quartz transducer: $\nu=95 \mathrm{kHz}, T=1.8$ and $2.5 \mathrm{~K}$.

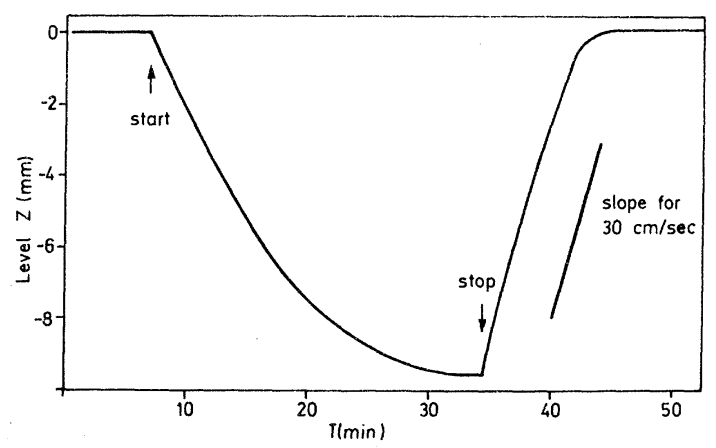

FIG. 5. Chart recording showing the liquid level $z$ in the capacitor of apparatus 1 (Fig. 2) as a function of time at $T=1.8 \mathrm{~K}$. The transducer was excited at $102 \mathrm{kHz}$ with an applied voltage of $6 V_{p p}$. The sound field created a level difference between the two baths. After the transducer was switched off, the liquid flowed back through the orifice $(10-\mu \mathrm{m}$ diam) at a velocity of about $30 \mathrm{~cm} / \mathrm{sec}$ until equilibrium between the liquid levels at $z=0$ was reached. No steps occurred.

locity of about $30 \mathrm{~cm} / \mathrm{sec}$ after switching off the transducer. The results at $T=1.3 \mathrm{~K}$ and $T=1.8 \mathrm{~K}$ were identical. We do not know why we could not see Josephson steps, if they exist in arrangements such as shown in Figs. 2 or 3.

\section{Flow Curves with "Free" Foil}

For some experiments with the simple setup shown in Fig. 3, we removed the 1-mm-thick plate to which the foil was glued. The foil was thus only fixed at its circumference and "free" everywhere else. We then obtained steplike flow through the 15- $\mu \mathrm{m}$-diam orifice in both flow directions when the sound field below the foil was switched on. The results for $T=1.8 \mathrm{~K}$ and $\nu=102 \mathrm{kHz}$ are shown in Fig. 6. ${ }^{10}$ The abscissa gives the level $z$ of the liquid in the glass capillary relative to an arbitrary mark. Emptying curves were obtained with a sound amplitude strong enough to pump liquid out of the capacitor. For filling curves, the transducer amplitude was reduced below the threshold for pumping. In both cases steps were observed. The complete arrest of liquid flow at discrete level differences of ten occurred for more than $15 \mathrm{~min}$. In order to kick the liquid out of these zero-flow states, we had to momentarily pulse the transducer amplitude (indicated by the spikes) or change the sound frequency (indicated by $f$ ). We could observe all the features of these steps which have been reported in Ref. 2.

In order to check the origin of the steps we changed the frequency of the ultrasound from 102 to $168 \mathrm{kHz}$. If we were observing the phenomenon which is called the "ac Josephson effect" in the literature, then the spacing between the levels of stability should have increased proportional to the 


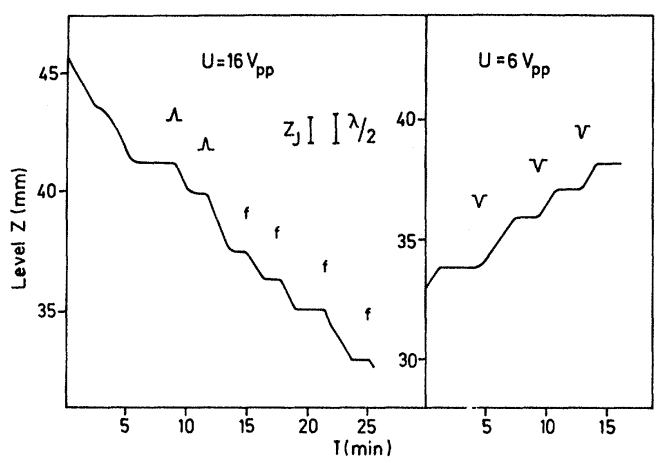

FIG. 6. Chart recording showing the time dependence of the liquid level $z$ in the glass capillary of apparatus 2 (Fig. 3) after removal of the plate to which the foil was glued. The level was measured relative to an arbitrary point on the capillary. The first trace shows the pumping action of the transducer $\left(\nu=102 \mathrm{kHz}, U=16 V_{\mathrm{pp}}\right)$. For the backflow shown in the second trace, the voltage at the transducer was reduced to $6 V_{\text {pp }}$ for a vanishing pumping action. The liquid was kicked out of the zero-flow states by increasing the transducer power (spikes) or changing its frequency $(f)$ momentarily. The ins ert shows the expected step sizes for the ac Josephson effect $\left(z_{j}\right)$ as well as for a half-wavelength of first sound in He II $(\lambda / 2)$. Several stable states with a spacing of $\lambda / 2$ are seen. $T=1.8 \mathrm{~K}$.

frequency from about 1 to $1.7 \mathrm{~mm}$ according to Eq. (1). The experimental results actually show a decrease of the spacing to $0.69 \mathrm{~mm}$ (see Fig. 7). That means that the step height was not proportional to $\nu$ but proportional to $\nu^{-1}$. Our results and

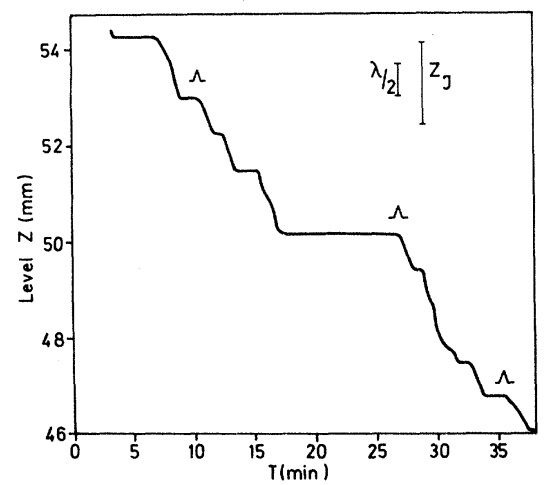

FIG: 7. Chart recording showing the time dependence of the liquid level $z$ in the glass capillary of apparatus 2 (Fig. 3) after removal of the plate to which the foil was glued. The level was measured relative to an arbitrary point on the capillary. The transducer was excited with $16 V_{p \mathfrak{p}}$ at $\nu=168 \mathrm{kHz}$. The liquid was kicked out of some of the stable states of zero flow by increasing the transducer power (spikes) momentarily. In addition, the expected step heights for the ac Josephson effect $\left(z_{J}\right)$ and for a half-wavelength of first sound in $\mathrm{He}$ II $(\lambda / 2)$ are shown. Several stable states with a spacing of $\lambda / 2$ are seen. $T=1.8 \mathrm{~K}$.
TABLE I. Comparison of the results observed at two frequencies with the theoretical predictions.

\begin{tabular}{cccc}
\hline $\begin{array}{c}\text { Frequency } \\
(\mathrm{kHz})\end{array}$ & $\begin{array}{c}\text { Observed steps } \\
(\mathrm{mm})\end{array}$ & $\begin{array}{c}\lambda / 2 \text { of first sound } \\
(\mathrm{mm})\end{array}$ & $\begin{array}{c}\text { Josephson effect } \\
(\mathrm{mm})\end{array}$ \\
\hline 102 & $1.15 \pm 2 \%$ & 1.14 & 1.03 \\
168 & $0.69 \pm 2 \%$ & 0.69 & 1.70 \\
\hline \hline
\end{tabular}

the theoretical predictions are summarized in Table I. The close agreement between the observed heights of the steps and half a wavelength of first sound in Heir led us to interpret our observations as stable states resulting from a series of ultrasonic standing waves in the liquid above the foil. The successive steps are associated with the changing helium level in the capillary. In this case the spacing between the states is proportional to $\nu^{-1}$ instead of being proportional to $\nu$ as for the ac Josephson effect. This origin for the steps in the flow was already mentioned in Refs. 1 and 2 but excluded by the authors as an explanation for their results. Our interpretation was further substantiated by the following fact. As mentioned above, the liquid in the main Dewar served as the outer superfluid bath for this setup. During the time of arrest of the flow, which was, for example, $10 \mathrm{~min}$ for the fourth state in Fig. 7, the liquid level in the main Dewar fell about $2 \mathrm{~mm}$ due to evaporation. Therefore our result means that the change of the liquid level in the outer chamber by several millimeters did not influence the level in the inner chamber when the flow had stopped. This emphasizes that the stable states with zero flow rate occurred at a particular level of the liquid in the inner chamber above the foil. They do not depend on the difference $\Delta z$ between the two liquid levels, as would be necessary to explain them by the ac Josephson effect.

In addition, we did not observe a shift in the position of the stable zero-flow state when a small temperature difference in the two chambers was generated. A temperature difference gives an additional contribution to the chemical potential diference between the superfluid baths. ${ }^{5}$

All of our observations are in agreement with the explanation that the interruption of flow results from ultrasonic standing waves above the foil and has no relation to the ac Josephson effect. The more complex geometry of apparatus 1 shown in Fig. 2 may have complicated the excitation of standing waves even after removing the backing plate from the foil. If they are excited and would result in a steplike flow, then the length of the liquid column does not necessarily have to be proportional to $n \lambda / 2$ due to the partly annular geometry.

These standing waves may result in a high pressure and in highly turbulent flow near the foil which may "close" the hole. Apparatus 2 filled with acetone was used to demonstrate that intense ultrason- 
ic standing waves can be excited above the foil by the transducer below it. They were detected by observing bubbles created by cavitation in the standing-sound-wave resonances. We consider our results as a demonstration that one can obtain steplike flow of superfluid helium through a small orifice which can be explained by classical effects. This observation seems to be of great importance because of the fundamental role of the Josephson effect for the understanding of superfluid helium. ${ }^{4}$
${ }^{*}$ Work supported by the Deutsche Forschungsgemeinschaft.

${ }^{\dagger}$ Present address: Kernforschungsanlage Jülich, 517 Jülich, Germany.

${ }^{1}$ P. L. Richards and P. W. Anderson, Phys. Rev. Letters 14,540 (1965); P. L. Richards, Phys. Rev. A $\underline{2}$, 1532 (1970).

${ }^{2}$ B. M. Khorana and B. S. Chandrasekhar, Phys. Rev. Letters 18,230 (1967); B. M. Khorana, Phys. Rev. 185, 299 (1969).

${ }^{3}$ J. P. Hulin, C. Laroche, A. Libchaber, and B. Perrin, Phys. Rev. A $\underline{5}, 1830$ (1972).

${ }^{4}$ B. D. Josephson, Phys. Letters 1, 251 (1962); Advan. Phys. 14, 419 (1965); P. W. Anderson, Rev. Mod. Phys. 38, 298 (1966).

${ }^{5}$ B. M. Khorana and D. H. Douglass, Jr., in Proceeding of the Eleventh International Conference on Low Temperature Physics, edited by J. F. Allen, D. M. Finlayson, and D. M. McCall (University of St. Andrews Printing Department, St. Andrews, Scotland, 1969), Vol. 1.
${ }^{6}$ P. Leiderer, Diplomarbeit TH München, 1968 (unpublished).

${ }^{7} \mathrm{G}$. Schofield and T. M. Sanders, Jr. [private communication and thesis of G. Schofield (University of Michigan, Ann Arbor, Michigan)]; M. E. Banton (private communication); D. Musinski and D. H. Douglass, Jr., in Proceedings of the Thirteenth International Conference on Low Temperature Physics, Boulder, 1972 (unpublished).

${ }^{8}$ Discussion seminar on "the ac Josephson effect in He Ir" (D. H. Douglass, Jr. , B. M. Khorana, F. Pobell, P. L. Richards, and T. M. Sanders, Jr.), in Proceedings of the Thirteenth International Conference on Low Temperature Physics, Boulder, 1972 (unpublished).

${ }^{9} \mathrm{H}$. A. Notarys and J. Andelin, Bull. Am. Phys. Soc. 12 , 1131 (1967); G. E. Watson, in Proceedings of the Thirteenth International Conference on Low Temperature Physics, Boulder, 1972 (unpublished).

${ }^{10}$ The weak pumping action of the transducer for $T>T_{\lambda}$ prevented a performance of this experiment above $T_{\lambda}$.

\title{
Asymptotic Properties of Autocorrelation Functions and the Enskog Expansion in Three-Dimensional Simple Classical Fluids
}

\author{
Y. Pomeau \\ Laboratoire de Physique des Plasmas, Université Paris-Sud, Centre d'Orsay 91, France \\ (Received 5 June 1972)
}

The $t^{-3 / 2}$ long-time behavior of the Green's-Kubo autocorrelation functions is the first term in an infinite series of general order $t^{1 / 2^{n}-2}, n$ integer $\geq 1$. The coefficients of these series for the shear and bulk viscosity and for the heat conductivity are given in terms of linear recurrence relation. Similarly, after the usual Navier-Stokes order (in $k^{2}$ ), there exists an infinite expansion for the frequencies of the hydrodynamical modes with terms of general order $k^{3-1 / 2^{n}}$. The mean square displacement of a particle in a fluid is given, for large times, by an infinite series, the first term being the well-known Einstein displacement $6 D t$, and the following ones proportional to $t^{1 / 2^{n}}$. As an application of this expansion of the hydrodynamical theory beyond the Navier-Stokes order, the pressure pattern in a weak shock wave is computed.

\section{INTRODUCTION}

A number of methods lead to the conclusion that the Green's-Kubo (GK) autocorrelation functions decrease for large times like $t^{-3 / 2}$ in three-dimensional simple fluids ${ }^{1-5}$ and binary mixtures. ${ }^{6} \mathrm{Al}-$ though it cannot be considered that these methods provide a vigovous proof of this behavior, it does not appear unreasonable to have some confidence in this kind of result (and we shall proceed as if it was firmly grounded) owing particularly to the diversity of the methods of derivation: moleculardynamics computations, ${ }^{1}$ kinetic theory, ${ }^{2}$ generalized Landau-Placzeck theory, ${ }^{1,3,4}$ and, in the case of the Brownian problem, hydrodynamic computation. ${ }^{5}$ It will be shown in this paper that this $t^{-3 / 2}$ behavior is the beginning of infinite series of general order $t^{1 / 2^{n}-2}, n$ integer $\geqslant 1$.

In Sec. II the most salient features of an already published derivation ${ }^{4}$ of the $t^{-3 / 2}$ behavior are sum- 